\title{
Cardiovascular screening of Paralympic athletes reported by chief medical officers of the PyeongChang 2018 Paralympic Winter Games
}

\author{
Brett G Toresdahl, ${ }^{1}$ Cheri Blauwet, ${ }^{2,3}$ Cindy J Chang, ${ }^{4,5}$ Daphne I Ling, ${ }^{6}$ \\ Irfan M Asif ${ }^{7}$
}

The 2009 IOC Consensus Statement on Periodic Health Evaluation (PHE) of Elite Athletes recommends a 12-lead ECG, but the frequency of the PHE elements is not defined. ${ }^{1}$ In 2011, the International Paralympic Committee (IPC) approved the IPC Medical Code, which describes the need for routine PHE based on the latest medical knowledge. ${ }^{2}$ However, the components and frequency of screening are also not delineated. Recent research has evaluated the cardiovascular (CV) screening practices of Olympic athletes, but there are limited data regarding $\mathrm{CV}$ screening of Paralympic athletes. ${ }^{34}$

This study aimed to investigate current practices for CV screening of Paralympic athletes. Chief medical officers (CMOs) for National Paralympic Committees of the PyeongChang 2018 Paralympic Winter Games were identified by the IPC and surveyed regarding the $\mathrm{CV}$ screening practices of their respective teams. The primary outcome was the utilisation of $\mathrm{CV}$ screening components as part of the PHE. The secondary outcomes were the frequency of performing the CV screening components.

A total of 35 of the $49(71.4 \%)$ countries participating in the PyeongChang 2018 Paralympic Winter Games had a

${ }^{1}$ Primary Care Sports Medicine Service, Hospital for Special Surgery, New York City, New York, USA

${ }^{2}$ Department of Physical Medicine and Rehabilitation, Harvard Medical School, Spaulding Rehabilitation Hospital, Boston, Massachusetts, USA

${ }^{3}$ Physical Medicine and Rehabilitation, Brigham and Women's Hospital, Boston, Massachusetts, USA ${ }^{4}$ Department of Orthopaedic Surgery, University of California - San Francisco, San Francisco, California, USA

${ }^{5}$ Department of Family and Community Medicine, University of California - San Francisco, San Francisco, California, USA

${ }^{6}$ Sports Medicine and Shoulder Service, Hospital for Special Surgery, New York City, New York, USA 7 University of South Carolina School of Medicine Greenville, Department of Family Medicine, Greenville Health System, Greenville, South Carolina, USA

Correspondence to Dr Brett G Toresdahl, Primary Care Sports Medicine Service, Hospital for Special Surgery, New York City NY 10021, USA;

toresdahlb@hss.edu
CMO who could be identified. Of these, 26 completed the survey $(74.3 \%$ of identified CMOs), representing 399 of 567 (70.4\%) Paralympic athletes. Written mandates existed regarding the PHE for 17 of 26 responding countries (65.4\%). The following screening components were performed at least once in all or most athletes from responding countries: 25 (96.1\%) personal history, 24 (92.3\%) family history, $24(92.3 \%)$ physical exam, 22 (84.6\%) ECG, 9 (34.6\%) echocardiogram and $10(38.5 \%)$ stress test (table 1$)$. Twenty-two countries (84.6\%) screened all or most athletes at least once with personal history, family history, physical exam and ECG. For the two countries that reported screening few or no athletes with ECG, reasons for not using ECG were expense of screening for both countries, as well as lack of physicians to interpret ECG, lack of evidence about cost-effectiveness and lack of consensus about follow-up testing recommendations for one country.

Most countries (19 of 26, 73.1\%) performed CV screening with at least one component in all or most athletes on an annual basis. ECG screening was performed annually in 15 of the 22 countries $(68.1 \%)$ that use ECG. Four countries reported that impairment type influenced the PHE. Qualitative descriptions included modifying the stress test protocol, for example, arm ergometry rather than treadmill, or refraining from performing a stress test depending on the athlete's degree of impairment, for example, athletes with high spinal cord injury (SCI) or severe cerebral palsy. No CMO reported performing additional or more frequent CV screening based on an athlete's impairment type.

This is the first study to assess CV screening practices among elite Paralympic athletes. Strengths of the study include that the responding CMOs represented over $70 \%$ of athletes, while limitations include the inability to link screening outcomes to CV screening practices. Nevertheless, important findings include the fact that $85 \%$ of the responding countries screen the majority of their athletes with personal history, family history, physical exam and ECG, and nearly $70 \%$ of these countries perform all four components on an annual basis. This finding may reflect the increased risk for CV disease in Paralympic athletes, possibly attributable to higher prevalence of CV disorders, structural cardiac adaptations that result from haemodynamic alterations in spinal cord-injured or amputee athletes, or increased risk of coronary disease due to a relative decrease in overall physical activity. ${ }^{5-7}$ The proportion of Paralympic athletes screened for CV disease is higher than Olympic athletes, with $92.3 \%$ vs $79.8 \%$ screened with personal history, family history and physical exam, as well as $84.6 \%$ vs $74.5 \%$ with ECG. ${ }^{4}$ Furthermore, athletes with SCI may have difficulty 
describing symptoms of angina due to decreased sensory feedback.

Paralympic athletes are a distinct population with unique $\mathrm{CV}$ demands and physiology. Screening for diseases that predispose these athletes to sudden cardiac death (SCD) should include an individualised approach based on estimated risk for SCD and available resources. Further research is needed to better understand optimal screening strategies for this cohort of athletes.

Acknowledgements The authors wish to thank the International Paralympic Committee (IPC) and IPC Medical and Scientific Director Dr Peter Van de Vliet, as well as the IPC Medical and Sports Science Committees, for their support of this study.

Contributors BGT: concept, design, acquisition of data, analysis of data, interpretation of data, drafting the manuscript, approval of final version. CB: design, acquisition of data, interpretation of data, critical revising, approval of final version. CJC: concept, design, drafting the manuscript, critical revising, approval of final version. DIL: analysis of data, interpretation of data, critical revising, approval of final version. IMA: concept, design, interpretation of data, drafting the manuscript, critical revising, approval of final version.
Competing interests None declared.

Patient consent Not required.

Ethics approval The study was approved by Hospital for Special Surgery Institutional Review Board on 5 May 2016 and renewed on 5 May 2018 (study ID 2015-691), and the International Paralympic Committee.

Provenance and peer review Not commissioned; externally peer reviewed.

Data sharing statement No additional data available.

(c) Author(s) (or their employer(s)) 2019. No commercial re-use. See rights and permissions. Published by BMJ.

\section{(A) Check for updates}

To cite Toresdahl BG, Blauwet $C_{\text {, Chang }} \mathrm{CJ}$, et al. Br J Sports Med 2019;53:43-44.

Accepted 28 August 2018

Published Online First 15 September 2018

\section{CLlinked}

http://dx.doi.org/10.1136/bjsports-2018-099795

Br J Sports Med 2019;53:43-44

doi:10.1136/bjsports-2018-099708

\section{REFERENCES}

1 Ljungqvist A, Jenoure P, Engebretsen L, et al. The International Olympic Committee (IOC)

Consensus Statement on periodic health evaluation of elite athletes March 2009. Br I Sports Med 2009;43:631-43.

2 International paralympic committee medical code: international paralympic committee. 2011. https:// www.paralympic.org/sites/default/files/document/ 120131082554885_IPC\%2BMedical\%2BCode_Final. pdf (cited 3 Sep 2017).

3 Toresdahl BG, Chang C, Confino J, et al. Cardiovascular screening practices in US National Governing Bodies and National Paralympic Committees. Br J Sports Med 2017;51:1639-40.

4 Toresdahl BG, Asif IM, Rodeo SA, et al. Cardiovascular screening of Olympic athletes reported by chief medical officers of the Rio 2016 Olympic Games. Br I Sports Med 2018:52:1097-100.

5 Pelliccia A, Quattrini FM, Squeo MR, et al. Cardiovascular diseases in Paralympic athletes. $\mathrm{Br} J$ Sports Med 2016;50:1075-80.

6 Myers J, Lee M, Kiratli J. Cardiovascular disease in spinal cord injury: an overview of prevalence, risk, evaluation, and management. Am J Phys Med Rehabil 2007:86:142-52.

7 Naschitz JE, Lenger R. Why traumatic leg amputees are at increased risk for cardiovascular diseases. QJM 2008;101:251-9. 